\title{
Strategic Deployment of Network Monitors for Attack Attribution
}

\author{
Invited Paper \\ Young June Pyun and Douglas S. Reeves \\ Department of Computer Science \\ North Carolina State University, Raleigh, NC 27695, USA \\ Emails: \{yjpyun, reeves\}@ncsu.edu
}

\begin{abstract}
Attacks launched over the Internet have become a pressing problem. Attackers make use of a variety of techniques to anonymize their traffic, in order to escape detection and prosecution. Despite much research on attack attribution, there has been little work on optimizing the number and placement of monitoring points for identifying the source of attacks with minimum ambiguity. This paper proposes such a method. The approach is based on the concept of graph separators. A separator partitions a network, such that the size of the separator is the number of monitors needed, and the size of a partition is the ambiguity in isolating the specific source of an attack. To achieve a desired degree of ambiguity, a good separator for the Internet is sought. Both vertex and edge separator heuristics are presented, which greedily select vertices of highest/lowest degree as monitors. The methods are evaluated for the Internet autonomous system (AS) topology. Experimental results show that the vertex separator heuristic requires just $5 \%$ of the ASes to be monitored to identify the source of an attack with little ambiguity. If only those links actually used for routing to a specific destination are considered, use of an edge separator requires $30 \%$ of the links to be monitored to achieve similar results. The results can be further improved if it is known that ASes have unequal probabilities of being the source of an attack.
\end{abstract}

\section{INTRODUCTION}

Network based attacks are a serious threat to the safety of the Internet. Despite efforts to protect the network infrastructure and networked computer systems, attacks continue to be launched and do substantial damage. Those who are charged with defending networked assets from the attacks would very much like to be able to identify the source of each attack so that appropriate action can be taken. Attack tracing and attribution is important for several reasons: 1) traceback of the attacks allows the Internet community to hold the attackers accountable, 2) knowledge of the attackers and their attack paths allows network administrators to better defend their systems, and 3) the existence of strong attribution will deter attackers from launching attacks. Once the source of an attack is identified, actions such as legal prosecution, termination of access, or other measures may be taken.

Unfortunately, identifying the attackers is a challenging task due to the anonymous nature of the Internet [1], lack of an

This work was supported by DTO under contract NBCHC030142. The contents of this paper do not necessarily reflect the position or the policies of the U.S. Government. attribution infrastructure on the Internet, and deliberate evasion techniques available to the attackers. These include address spoofing [2], packet encryption [3], [4], traffic camouflaging [5], anonymizing networks [6], stepping stones [7], [8], etc. Substantial research on attack attribution has been undertaken to overcome such evasive techniques. Several methods of IP traceback [9]-[11] have been proposed to find the source of an attack, even when source address spoofing has been used. Other work has targeted tracing a chain of connections through stepping stones (intermediate host computers) [7], [8], [12], [13]. In addition, connections through anonymizing networks have also been studied in recent work [14].

Generally, the structure of the network plays an important role in the above methods of attack attribution. Specifically, the Internet is composed of Autonomous Systems (ASes) where each AS normally corresponds to a single independent administration, such as an ISP. ASes are connected either directly to each other or at peering points, such as the Internet Exchange Points (IXPs) [15]. Routing between ASes is handled by BGP4 [16], an exterior gateway routing protocol. Attack attribution can be organized to operate at two levels. The first, or inter-AS level, is responsible for identifying the source AS of an attack. The second, or intra-AS level, is responsible for identifying the specific host within that AS that is responsible for the attack.

Most methods of attack attribution require some form of support from computer hosts and/or routers. This support may be necessary for passive observation of login attempts or network traffic, or for active modification of packet headers, contents, or timing. Network elements that provide such support for attack attribution are referred to as network monitors and may either be routers with enhanced capabilities or hardware specialized just for this function. Minimizing the required support is an important goal for the sake of cost, deployment effort, and lack of cooperation by network operators. Despite considerable research on attack attribution, little attention has been paid to minimizing the number and optimizing the placement of monitors in a network generally, or across the Internet specifically. If monitoring capabilities are not universally available, it is important to pick monitoring points carefully. A poor selection may result in limited ability to isolate the source of an attack, or in substantial overdeployment of monitors. 
There is no single entity that governs this type of issue for the Internet; the Internet is operated as a cooperative enterprise, with independent policies and regulations. In some circumstances, such as security, we believe there may be enough common interest to motivate shared or cooperative oversight. The proposed approach does not require ceding control over network administration; it only presumes network operators are willing to share information for defensive purposes, and will want to do so as economically as possible. It is not realistic to expect universal cooperation, in which case, the achievable degree of precision for a given level of available monitoring capability is an important question to answer.

This paper proposes a new strategy for deploying network monitors for attack attribution. It focuses on the number and placement of the monitors while measuring the ambiguity in identifying the source of an attack. The problem is transformed into a graph partitioning problem, which is solved by finding a separator that divides the graph into partitions of sufficiently small sizes. In this transformed problem, the size and membership of the separator corresponds to the number and locations of the monitors, and the size of each partition corresponds to the ambiguity in identifying the specific source of an attack. To achieve a desired degree of ambiguity with minimal cost, a good separator for the Internet topology is sought. Two basic heuristics are proposed which greedily select the highest degree (for node monitoring purposes) or the lowest degree (for link monitoring purposes ) AS from the largest partition, where the degree of an AS is the number of peers it has. A hybrid method combining both types of separators is also proposed. In addition to these general approaches, exploiting special information, such as interest in a single destination (target) AS, and unequal probabilities of ASes being the attack source, are addressed as well.

The expected benefit of the proposed approach is the ability to determine a minimal number and close-to-optimal placement of monitors in order to achieve a desired level of accuracy in identifying the source of an attack. Doing so will speed up the deployment process, ease the burden of administration, and potentially speed up the process of attack tracing as well. All of these benefits are worthwhile, due to the complexity and size of the Internet.

The remainder of this paper is organized as follows. Section 2 defines a theoretical model for the problem and introduces possible general approaches. Section 3 proposes new methods for deploying network monitors for the Internet and section 4 evaluates them through experiments. Then, section 5 reviews the previous work on monitor deployment. Finally, section 6 summarizes this paper with a conclusion and future work.

\section{Problem Statement}

The Internet topology can be modeled as an undirected graph $G(V, E)$, where the vertex set $V$ represents the set of Autonomous Systems (ASes) and the edge set $E$ represents the set of links between ASes. Given a graph $G$, a separator $S$ is a set of vertices or edges in $G$ that partitions $G$ in some manner [17], [18]. There are two choices of the separator;
- A vertex-separator $S_{V}$ produces

$$
\begin{gathered}
V=S_{V} \cup P_{1} \cup \cdots \cup P_{k}, \\
\text { where } P_{i} \cap S_{V}=\emptyset \text { and } P_{i} \cap P_{j}=\emptyset \text { for } i \neq j,
\end{gathered}
$$

and the boundary of each $P_{i}$

$$
\partial P_{i}=\left\{u \mid u \notin P_{i} \text { and }(v, u) \in E \text { for some } v \in P_{i}\right\},
$$

is a subset of the separator $S_{V}$.

- An edge-separator $S_{E}$ produces

$$
V=P_{1} \cup \cdots \cup P_{k} \text {, where } P_{i} \cap P_{j}=\emptyset \text { for } i \neq j,
$$

and an edge-separator

$$
S_{E}=\left\{(u, v) \mid u \in P_{i}, v \in P_{j}, i \neq j\right\} .
$$

That is, $S$ (either $S_{V}$ or $S_{E}$ ) partitions $G$ into $k$ components $C_{i}$, where $C_{i}$ is a maximal connected subgraph of $G$ induced by $P_{i},|S|$ is the number of vertices/edges in $S$, and $\left|P_{i}\right|$ is the number of vertices in $C_{i}$.

Let $P$ represent the set of all partitions produced by the separator. That is, $P_{E}=\left\{P_{1}, \cdots, P_{k}\right\}$ for the edge separator and $P_{V}=\left\{P_{1}, \cdots, P_{k}\right\} \cup S_{V}$ for the vertex separator. Then, when considering the edge separator, the maximum partition size is defined simply as $\max \left\{\left|P_{i}\right|\right\}$, and the average partition size is

$$
\sum_{i=1}^{k}\left|P_{i}\right| / k
$$

For the vertex separator, the maximum partition size is defined in the same way, but the average partition size is

$$
\left(\left|S_{V}\right|+\sum_{i=1}^{k}\left|P_{i}\right|\right) /\left(\left|S_{V}\right|+k\right)
$$

where each vertex in the vertex separator is considered to be a partition of size 1 .

We define a monitor placement problem for the Internet as finding a separator $S$ for a graph $G$ (representing the Internet) which divides $G$ into partitions $P$, such that the maximum or average partition size is minimized for a given separator size $|S|$. An alternative version of the problem is to minimize the separator size, given a required maximum or average partition size.

The motivation for this problem is as follows. The separator represents the set of ASes (vertex separator) or links (edge separator) which are selected for monitoring. Its size translates into the cost of monitor deployment and is bounded or minimized for the practical reasons stated in the previous section. On the other hand, the partition size is our measure of performance, as it captures the ambiguity of attack attribution. The source of an attack which is traced to a particular partition can be narrowed down to one of the ASes in that partition. Links between those ASes, and the ASes themselves, are not monitored, so a more precise identification is not possible without further action. The attack cannot have originated from an AS in another partition, since all traffic between partitions passes through at least one of the monitors. Fig. 1 illustrates an 


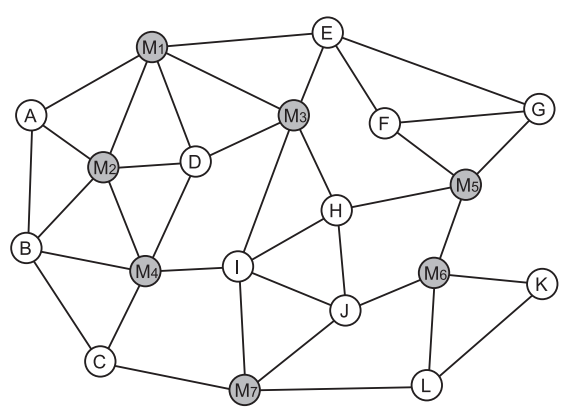

(a) Original graph with separating vertices.

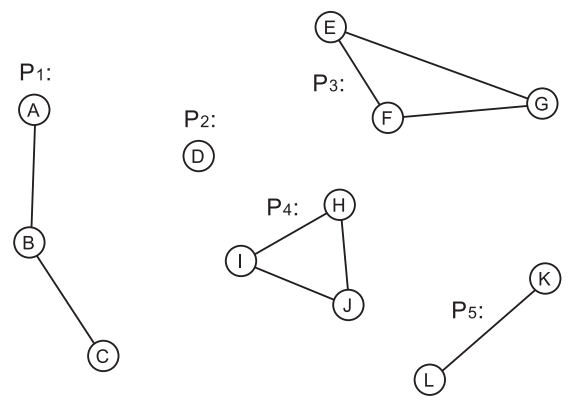

(b) Graph after vertex separation.

Fig. 1. Illustration of graph partitioning with a vertex separator.

example how a vertex separator $\left\{M_{1} \cdots M_{7}\right\}$ divides a graph with 19 vertices into small partitions $\left\{P_{1}, P_{2}, P_{3}, P_{4}, P_{5}\right\}$. In this configuration, the maximum ambiguity in identifying the source of an attack is 3 , where the possible ambiguity ranges from 1 to 19. Note that the ASes which are monitored are not removed from the Internet; they are simply instrumented for attack identification, and may themselves be the source of an attack.

It is possible for the monitor placement problem to be defined with optimization metrics other than the ambiguity of attribution. As an example, a possible metric would be the distance from a monitor to the source of an attack. The resulting problem is referred to as a p-center problem [19]. A p-center of a graph $G$ is a set $S_{p}$ (where $\left|S_{p}\right|=p$ ) if the maximum distance from every vertex not in $S_{p}$ to $S_{p}$ is the minimum. Unfortunately, this approach would not be suitable for purposes of attack attribution, as attack paths are not guaranteed to pass through at least one member of the pcenter. Another possible metric would be maximizing the path coverage of the monitors, which is the fraction of the links that are monitored. This metric also does not target identification of an attack source. We believe that the proposed metric of ambiguity, based on partition size, better captures the desired outcome.

In this paper we investigated only the issue of monitor placement for the Internet. (The techniques may be useful as well for smaller networks, but that issue has not been investigated in depth.) The distinct topological properties of the Internet are therefore relevant. This paper uses for experimentation purposes a particular Internet topology, which is a snapshot

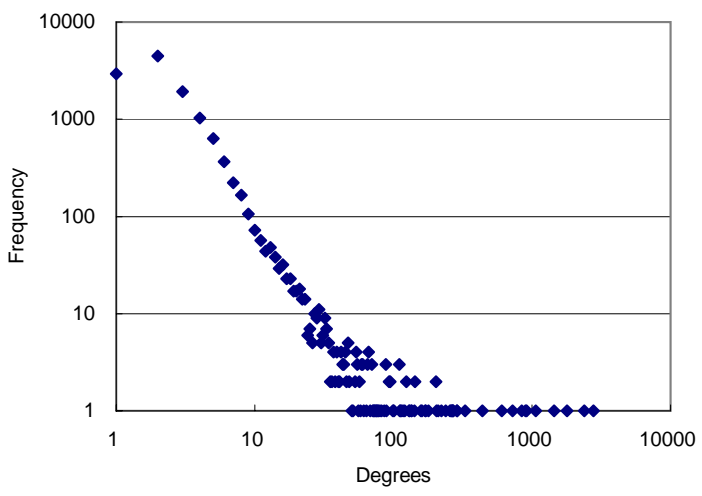

Fig. 2. Degree histogram of Autonomous Systems on a AS-level Internet topology with 12,538 ASes and 32,932 links. Data collected on August 2003 with skitter by CAIDA.

of an AS-level graph collected at CAIDA [20], [21]. It was generated by mapping routable IP prefixes (collected through traceroute-like network probing) to the ASes responsible for routing them. This was based on BGP routing tables collected by the University of Oregon's RouteViews project [22]. The resulting graph consisted of 12,538 AS nodes and 32,932 peering sessions (links), with a maximum AS degree of 2,766. Fig. 2 shows a degree histogram of ASes in this Internet. This graph exhibits power-law connectivity, a typical structural characteristic of the Internet described in [23].

\section{Monitor Deployment Strategy}

Finding an optimal separator for arbitrary graphs is an intractable problem. Instead, heuristics have been used to obtain acceptable non-optimal solutions in reasonable running time. These include the well-known heuristic by Kernighan and Lin [24] and a more advanced multilevel algorithm by Karypis and Kumar [25] used in the METIS software. These heuristics are generally accepted as being high-quality methods in practice. Unfortunately, they are not well-suited for solution of the monitor placement problem, as their assumptions and optimization goals are slightly different than is required for optimization of monitor placement. Their objective is to partition a given graph into a specified number of subgraphs of roughly equal sizes, while optimizing the separator size. In contrast, for the monitor placement problem, it is the partition sizes (maximum or average), as well as the separator size, that are of primary interest.

The following sections present approximation methods that can be used to solve the separator problem for the Internet. In addition to the basic monitor placement problem, this paper also addresses several restricted (but realistic) versions of the problem where the resulting partition sizes can be further reduced for the same cost. These versions consider restrictions on the potential target of an attack and on the potential sources of an attack.

\section{A. Vertex Separator}

A vertex separator divides a graph into partitions by removing the vertices of the separator from the graph along with 
the edges adjacent to those vertices. For purposes of monitor placement, a vertex separator selects a subset of ASes on the Internet as monitoring points, assuming all links connecting those ASes to their peers will therefore be monitored. Every AS not selected for monitoring will be in one of the partitions of ASes. The partition size reflects the ambiguity in being able to trace attacks specifically to that AS. The cost of monitor deployment, using a vertex separator, will be interpreted as the number of ASes selected to monitor (i.e., the size of the separator). ${ }^{1}$

An optimal solution for a vertex separator would be a bruteforce method that, given a graph $G(V, E)$ and a separator size $s$, tries every possible combination of $s$ vertices in $G$ and chooses the one that minimizes the maximum partition size. This approach would require $O\left(|V|^{s}\right)$ time, and is therefore infeasible for application to large networks, such as the Internet. A heuristic approach is hence proposed based on the following insights. Increasing the number of partitions for a fixed size separator will produce smaller partitions. Removing (monitoring) higher-degree vertices will create more partitions than removing low-degree vertices. Note that the Internet topology follows the power-law distribution, where a small fraction of the ASes accounts for most of the links in the network. Therefore, our vertex separator uses a greedy approach, which selects the highest degree vertex in the largest partition of the graph at each step in the process. The algorithm is as follows.

Approximation Algorithm for a Vertex Separator:

1) Select a largest partition $P_{m}$ with ties broken randomly.

2) Select a highest-degree vertex $v$ in $P_{m}$ with ties broken randomly.

3) Remove $v$ from $P_{m}$, along with its edges, and add $v$ to the separator $S$.

4) Repeat steps 1-3 until desired $|S|$ or $|P|$ is achieved.

As a result of selecting high-degree nodes, two adjacent high-degree ASes may be selected as monitoring points. If this occurs, a connecting link between the two ASes will be monitored at both ends. Since the optimization goal is to minimize the number of monitored vertexes, eliminating such redundant coverage of links is not considered by the algorithm.

\section{B. Edge Separator}

An edge separator divides a graph into partitions by removing edges from the graph (and adding them to the separator). In terms of monitor placement, an edge separator selects a subset of links between ASes on the Internet for monitoring, assuming each link is monitored separately, independent of the ASes to which they are attached. Using the edge separator approach, each AS will be in a partition, which is surrounded by links that are monitored. The cost of monitor deployment, using an edge separator, is measured by the number of links selected for monitoring.

\footnotetext{
${ }^{1}$ Although this paper abstracts the Internet into a macro-scopic topology and hence measures the cost in units of ASes, the real cost should reflect the number of peering links attached to the monitoring ASes.
}

Here, the above vertex separator is modified to solve the edge separator problem for the Internet. Rather than selecting the highest-degree vertex, the lowest-degree vertex is used for the edge separator. This choice provides a faster breakdown of the graph, efficiently producing smaller partitions. The algorithm is as follows.

Approximation Algorithm for an Edge Separator:

1) Select a largest partition $P_{m}$ with ties broken randomly.

2) Select a lowest-degree vertex $v$ in $P_{m}$ with ties broken randomly.

3) Remove every edge $e$ of $v$ from $P_{m}$, and add $e$ to the separator $S$.

4) Repeat steps 1-3 until desired $|S|$ or $|P|$ is achieved.

The major difference between these two versions of separators is the assignment of cost. In the vertex separator, cost is computed as the number of ASes which are monitored, while in the edge separator, cost is calculated as a function of the edges that must be monitored. Since the actual method of monitoring is unknown, we cannot state clearly which of these two is more important and realistic. It may be that AS monitoring (vertex separator) requires a higher degree of access or cooperation than link monitoring (edge separator), but the cost per link may be less than without such cooperation. Therefore, results for both are included.

\section{Hybrid Method}

There are advantages to both types of separators. AS monitoring is potentially more comprehensive and cost effective, but requires more effort (and a higher degree of cooperation) on the part of the AS administrators. We therefore investigated a hybrid method, in which only a small number of ASes are directly monitored, combined with a much larger number of links monitored.

The hybrid method works as follows. A few vertices of highest degree are selected and added to the separator, along with their attached edges. ${ }^{2}$ A reduced graph results from the removal of these vertices and edges. An edge separator is then applied to the reduced graph to select individual edges for monitoring.

\section{Single Destination Method}

The problem as stated assumes that every AS on the Internet is equally likely to be the target of an attack. Under this assumption, all paths through the Internet from any source AS to any destination AS must be considered possible. In some circumstances it may be that only specific targets (destinations) are of interest to attackers, or worth the effort of defending. In the extreme cases, it may only be desired to attribute attacks on a single target or AS. Finding a separator for the all sources / all destinations case is likely to be highly inefficient at solving this specialized problem.

Our solution to this single destination problem incorporates the BGP routing information as well as the network topology.

\footnotetext{
${ }^{2}$ Other heuristics for picking the small set of monitored ASes can be imagined; this is the only choice that was investigated, however.
} 
The motivation for doing this is to exclude from consideration those links that are not on the routing path from any source to that destination. For each destination AS, the BGP routing information can be used to construct a routing tree, rooted at that destination, from all ASes on the Internet. Only the links in this tree need to be considered for monitoring attacks. ${ }^{3}$

Assuming a tree $T$ rooted at a given vertex $d$, an edge separator is used on $T$ for partitioning it and selecting links to monitor. The edge separator, given a pre-defined maximum partition size $p$, works as follows.

Approximated Edge Separator for Sink Trees:

1) Perform a depth-first-search (DFS) on $T$, starting from $d$, with the children of a vertex selected in random order.

2) At each step of the depth-first search, construct a subtree consisting of vertices that have been visited.

3 ) If a new subtree would exceed $p$, prune the subtree of the previous step, adding the removed edge to the separator.

4) Repeat the process until the root is reached.

At the end of this process, the separator $S_{E}$ consists of those edges that are pruned during the process. $T$ is partitioned by $S_{E}$ into subtrees $T_{i}$ of size $\left|T_{i}\right| \leqslant p$.

\section{E. Unequal Probabilities Attack Source Problem}

The general problem also assumes that every AS on the Internet is equally likely to be the source of an attack. In some circumstances it may be that some networks are more likely to be the source of an attack than others. Use of this information can lead to a more efficient solution of the monitor placement problem. $^{4}$

A weighted separator is proposed for this version of the monitor placement problem. The weights are assigned to ASes according to their likelihood of being an attack source. The proposed separator heuristics (both vertex- and edge-based) are modified as follows. At each iteration of the algorithm, a new AS is selected from a partition with the highest aggregate attack probability. The selection of the monitors from the partition is unchanged. Starting from a single partition with an aggregate attack probability of $100 \%$, this process divides a network into partitions whose aggregate attack probability represents the ambiguity in identifying the attacker as well as the probability of that AS being a source of a potential attack. Note that if all ASes are equally likely of being the attack source, the weighted separator produces the same result as the unweighted version.

A modified definition of partition size (or ambiguity) is given as follows, for comparison with the previous cases. Let $n=|G|$ be the number of vertices in graph $G$, and $p_{v}$ be the weight of vertex $v$ (i.e., the probability that the corresponding

\footnotetext{
${ }^{3}$ This approach is only useful for attacks that are routed using BGP. An attack which establishes an overlay (consisting, for instance, of stepping stones, or mixes), is able to do application-level routing. Links in the overlay that are not part of the BGP routing tree to the selected destination, will no be selected for monitoring by this method.

${ }^{4}$ It must be considered that attackers will exploit this assumption if they know monitoring is based on specific expectations. The gain in efficiency has to be weighed against this additional vulnerability.
}

AS will be the source of an attack). Then the weighted size of a partition $P_{i}$ is defined as

$$
n \sum_{v \in P_{i}} p_{v}
$$

That is, the ambiguity of the partition in the weighted case is equal to the number of vertices in a partition with equal total probability, for the case when all vertices are equally weighted. This allows the ambiguity between weighted and unweighted graphs to be compared, and will be used in the next section.

\section{EXPERIMENTAL RESULTS}

To evaluate the proposed heuristics, they were applied to a number of graphs, and the resulting performance and costs were measured. These graphs include an actual Internet topology, a reduced Internet graph, and a synthetic regular graph. The AS-level Internet topology [20], [21] described in section II was used in the first case, with 12,538 vertices and 32,932 edges. For the reduced version, this AS-level Internet topology was processed (using the proposed vertex separator) to obtain a partition consisting of 296 vertices connected by 1,067 edges; this was the second graph used for experimental purposes. This graph size was small enough to allow computation of the optimum solution, for comparison with the heuristic solutions. For the third graph, an almost 11regular graph consisting of 5,000 vertices and 27,424 edges was synthetically generated using a $\delta$-process [26]. This was designed to be a test case with a very different structure than the Internet, for evaluation of the heuristics under a variety of conditions. For the experiments investigating the single destination (target) problem, hypothetical shortest path routing trees were generated for the Internet topology, for randomly selected destinations.

For comparison with the proposed heuristics, we also present results for (a) separators which select vertices or edges at random, (b) an optimal vertex separator, and (c) a widely used edge separator (METIS). In each experiment, the maximum and average partition sizes were measured, as a function of the cost of the separator, for the sake of a trade-off analysis between ambiguity and cost. Results are plotted on semi-logscale graphs. For cases involving randomly-generated choices, sufficient tests were run until confidence intervals were less than $1 \%$ of the values measured; the intervals are therefore omitted from the graphs.

\section{A. Vertex Separator}

We evaluated the performance of the heuristic vertex separator on the actual Internet graph, and compared it to a method which randomly selected a set of vertices for the separator. The heuristic separator was also compared to the optimal vertex separator on the reduced graph described above. This provided insight into the quality of the heuristic, albeit on a graph of less than the full Internet topology. The optimal solution was implemented as described previously.

Fig. 3 shows that the heuristic vertex separator performs well, achieving small values for both maximum and average 

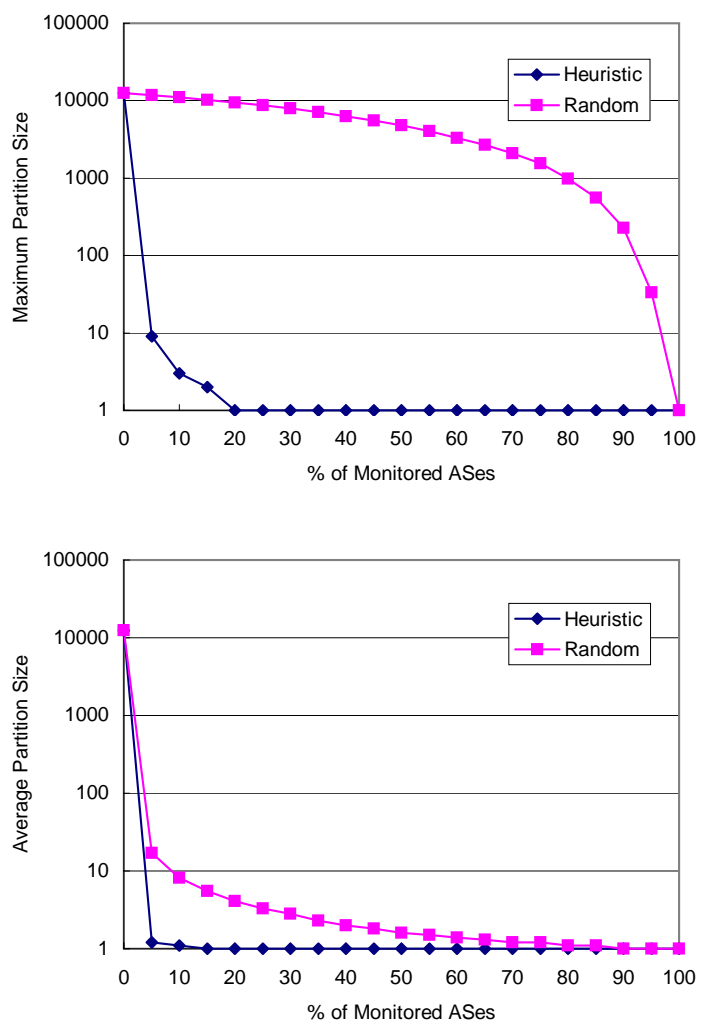

(b) Average partition size.

Fig. 3. Monitor placement by graph partitioning with a vertex separator Performance comparison of vertex separators for the Internet.

partition size on the Internet topology. With 5\% of the ASes selected as monitors, for instance, the maximum partition size is 9 and the average partition size is close to 1 . In this case, the source of an attack could be identified precisely with high probability. The performance is much superior to the random method, particularly with respect to the maximum partition size, by a factor of up to four orders of magnitude. The heuristic performs well due to the fact that a small number of ASes are highly connected in the Internet. Monitoring of these ASes quickly partitions the Internet graph, and attack attribution can be very effective. ${ }^{5}$

Comparison with the optimal solution (Fig. 4) shows that the proposed heuristic performs very close to optimal. The reduced graph with 296 vertices was used for this experiment, due to the unacceptably slow running time of the brute-force solution on the graph of the entire Internet topology.

\section{B. Edge Separator}

We next evaluated the performance of the heuristic edge separator on the actual Internet topology. Results were also computed for a method which randomly selected edges for the separator, and for METIS as well. METIS [25] is a widely used graph partitioning software package implementing an edge separator, based on a multilevel divide-and-conquer approach

\footnotetext{
${ }^{5}$ It does not escape our attention that attacking these ASes, if successful, would have a dramatic impact on Internet connectivity.
}
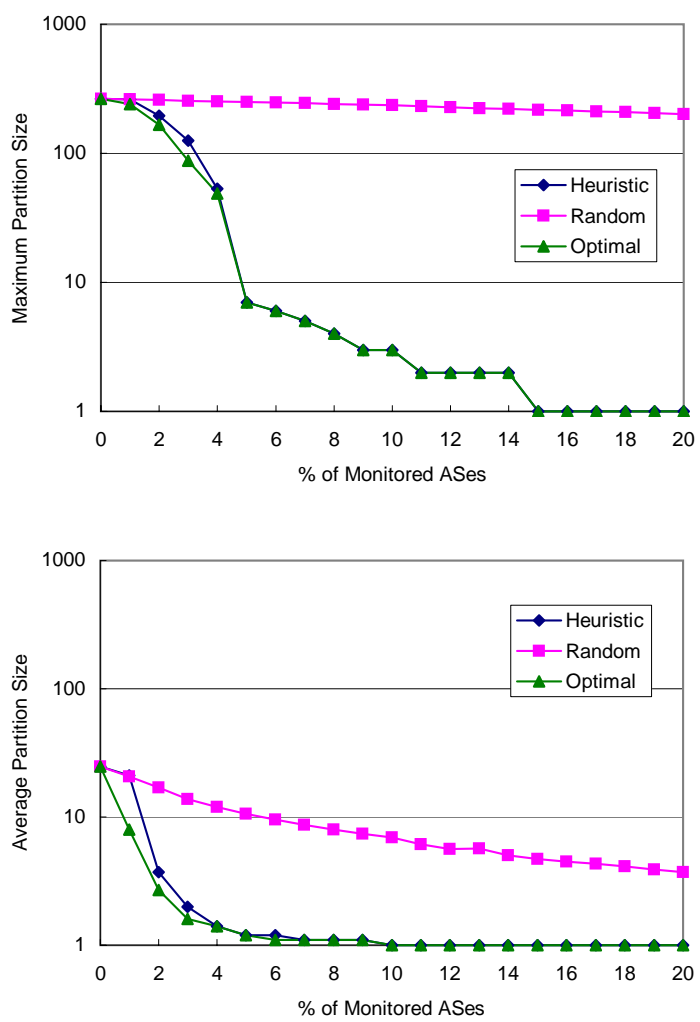

(b) Average partition size.

Fig. 4. Performance comparison of vertex separators for a reduced graph with 296 ASes.

using bisection. This method tries to produce equal-sized partitions while adding to the separator. These 3 methods were also evaluated on a synthetic regular graph, to demonstrate how the structural properties of the network topology affects the relative performance of the heuristics.

Fig. 5 shows the results of the experiment on the Internet topology. The graph shows that the costs of all separators are very high when the maximum partition size is the performance metric. Cost is much reduced for the proposed heuristic, although still substantial, when the average partition size is the performance metric. METIS produces the best results for the maximum partition size; METIS attempts to produce partitions of approximately equal size, which optimizes the maximum. However, it produces the worst result for the average partition size.

Fig. 6 shows the results of experimenting on a network with a topology that is almost regular. Both the heuristic edge separator and METIS produce similar results on this case as on the Internet topology, although the resulting partition sizes are higher. This difference confirms that the cost of monitor placement is influenced by the variability of degree distribution of the graph; regular graphs are more difficult to separate than power-law graphs.

We conclude that METIS is likely to produce a low cost edge separator for the Internet topology, when minimizing the maximum partition size is the goal. On the other hand, 

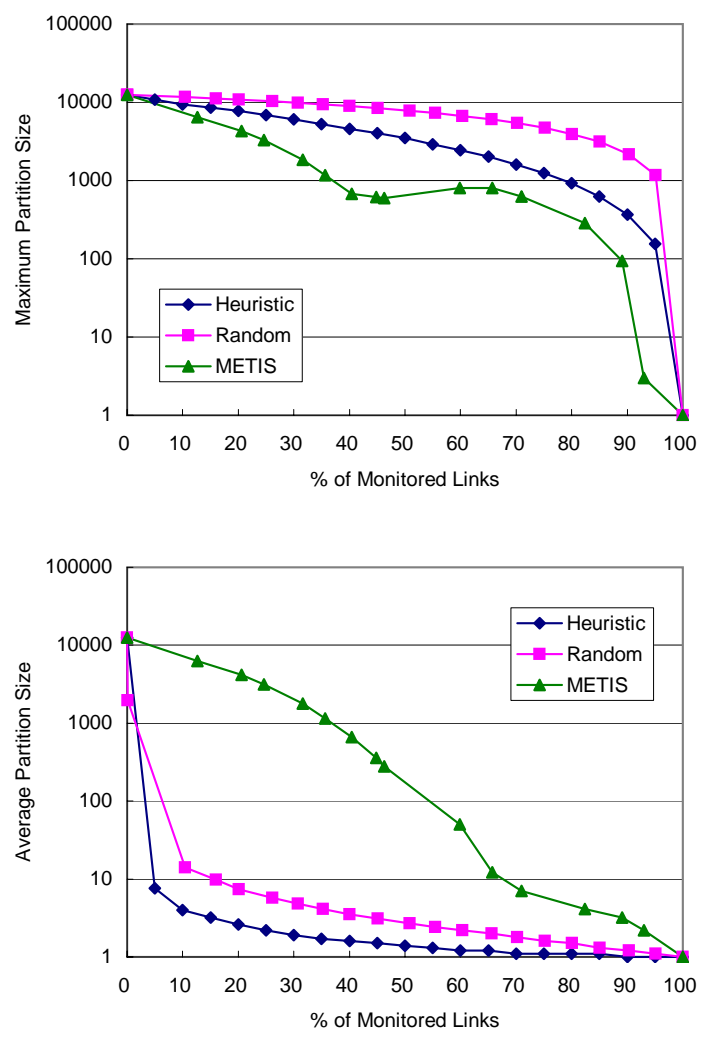

(b) Average partition size.

Fig. 5. Monitor placement by graph partitioning with an edge separator Performance comparison of edge separators for the Internet.

when the average partition size is the optimization metric, the heuristic edge separator performs much better.

\section{Hybrid Method}

This experiment evaluated the performance of the hybrid method on the Internet topology. Fig. 7(a) shows the maximum and average partition sizes as a function of the number of highest-degree ASes that are added to the separator. Fig. 7(b) shows the cumulative number of links incident to the highestdegree ASes. Based on this data, the top 5 ASes were selected for monitoring. These top 5 highest-degree ASes account for $29 \%$ of the total number of links on the Internet. With monitoring of just these ASes, the average partition size is 6, although the maximum partition size is still quite large.

After removal of these 5 vertices, the edge separator heuristic was applied to the resulting reduced graph; the results are shown in Fig. 8. This shows that the cost of the separator is decreased (conversely, the resulting partition size is reduced for the same number of edges monitored) when compared to the use of the edge separator alone (Fig. 5). With $10 \%$ of the edges removed, for instance, the maximum partition size is reduced by about $24 \%$, and the average partition size is reduced by about $43 \%$.

In a separate experiment (results not graphed due to space limitations) using Internet Exchange Points, we identified about 77 IXPs having at least 40 AS peers. If these IXPs
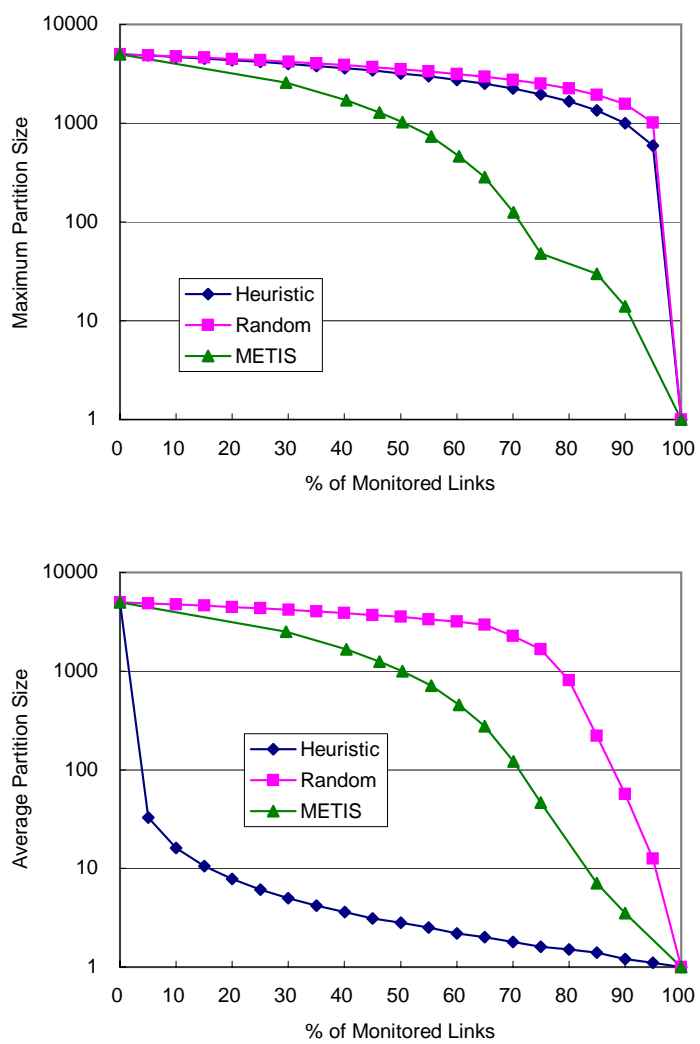

(b) Average partition size.

Fig. 6. Performance comparison of edge separators for a synthetic 11-regular graph with 5,000 vertices and 27,424 edges.

were monitored, the number of links in the Internet topology would be reduced by approximately $27 \%$. However, in this case, the results of edge separation following this reduction were not greatly improved (5\% or less improvement versus the pure edge-separator heuristic).

\section{Single Destination Problem}

This experiment evaluated the proposed method for the single destination (target) problem. This involves separating the graph consisting of the routing tree to a single destination. The motivation for examining this is to determine how much less expensive attack attribution is when protecting a single AS, rather than protecting all ASes on the Internet. Three separate experiments with ASes of varying degrees as the destinations were conducted. These ASes were of high (288), medium (10), and low (3) degree. A routing sink tree was generated for each of them, and then the edge separator heuristic for sink trees (described in section III-D) was applied to each.

Fig. 9 shows the result of the separator for the 3 different destinations. It shows that, for the single destination, the maximum partition size is well improved over the general case (Fig. 5). This improvement confirms the expectation that limiting the destinations to be protected using routing information will decrease the number of monitors required, or for a given number of monitors, decrease the ambiguity 

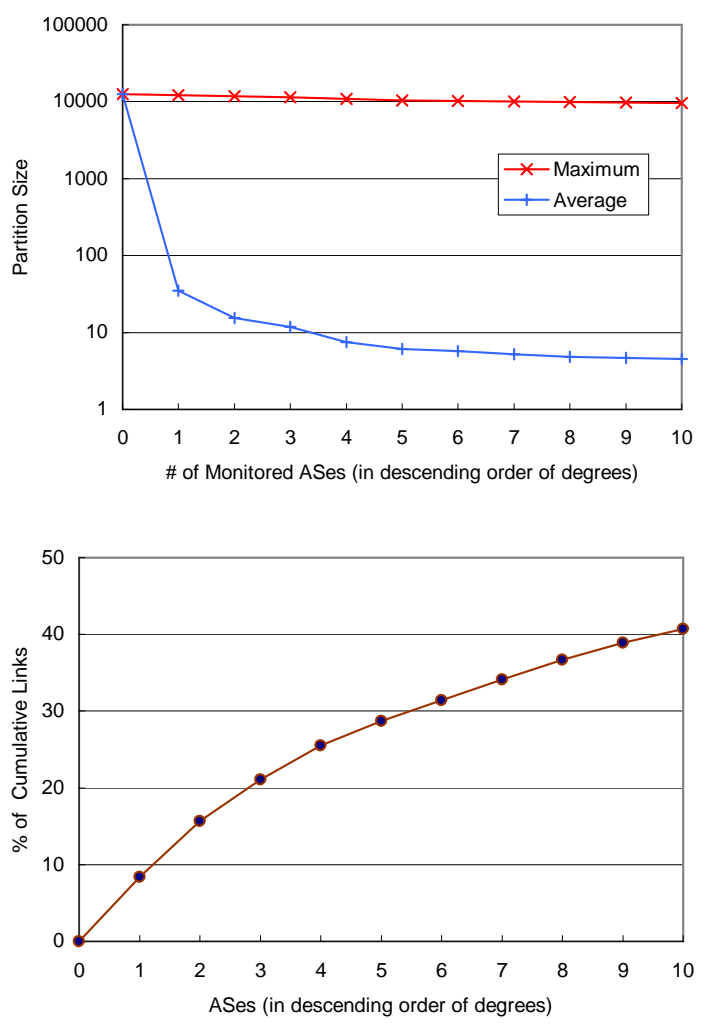

(b) Number of links attached.

Fig. 7. Vertex separator of top 10 highest-degree ASes.

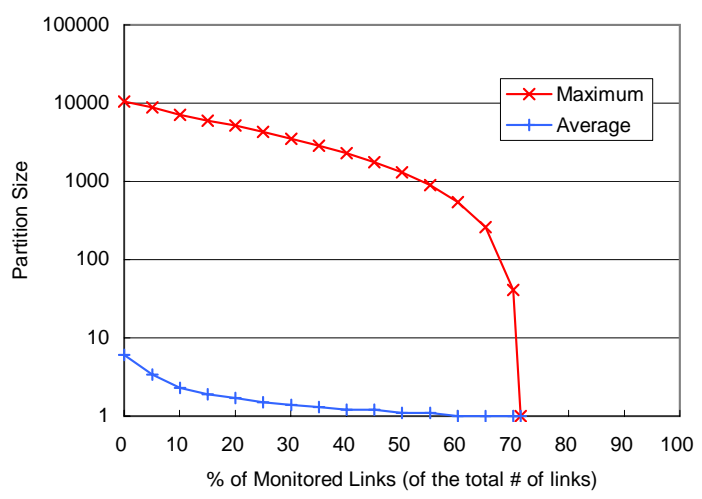

Fig. 8. Performance of hybrid separator for the Internet using edge separator after the removal of top 5 highest-degree ASes with vertex separator.

in attack attribution. However, the average partition size for the single destination case shows little improvement over the general case. This indicates that the proposed edge separator is efficient regardless of the number of ASes to protect, if the average ambiguity is to be minimized.

The results also show that there is not much difference in the maximum partition size results for the 3 types of destinations. The average partition sizes are identical for the following reason: a tree with $n$ vertices always has $n-1$ edges and removal of $k$ edges will always produce $k+1$ partitions, with
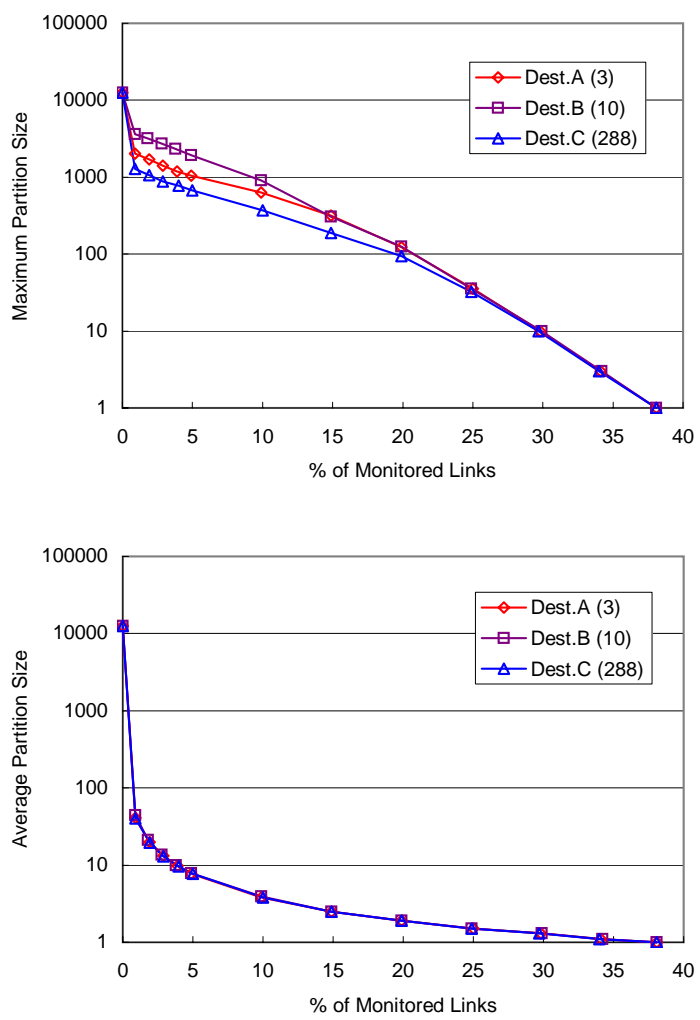

(b) Average partition size.

Fig. 9. Monitor placement for a single destination using routing information Performance comparison of 3 different destination ASes with varying degrees; Dest. A (degree=3), Dest. B (degree=10), and Dest. C (degree=228).

an average partition size of $n /(k+1)$.

\section{E. Unequal Probabilities Attack Source Problem}

Finally, weighted separators for the unequal probabilities attack source problem were evaluated. This experiment demonstrated the effect of exploiting knowledge of the attack source in the process of monitor deployment. For simplicity, we used a hypothetical attack probability assignment of ASes, into quartiles. That is, ASes were divided into four equalsized groups, where ASes in a same quartile had the same probability, and the probability of all ASes summed to $100 \%$. The aggregate attack probability of the fourth quartile was about $90 \%$, and the first 3 quartiles constituted $10 \%$ all together. Note that each AS was randomly assigned to a quartile with a specific individual probability as shown in Fig. 10.

The results using the weighted vertex separator are shown in Fig. 11(a) and the results using the weighted edge separator are shown in Fig. 11(b). Both are measured for the maximum and the average ambiguity of the fourth quartile. The results show that both types of separators benefit from the additional information (i.e., the unequal probabilities of ASes being the source of an attack). The maximum partition sizes in both cases were noticeably improved (about $70 \%$ reduction for both separators). For the average values, the improvements measured about $10 \%$ and $40 \%$ for vertex and edge separators, 


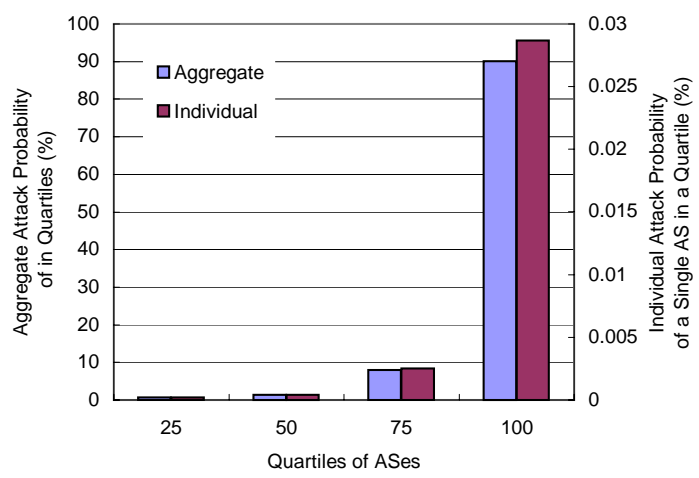

Fig. 10. A hypothetical attack probability assignment of ASes in quartiles. The aggregate attack probability of ASes in the fourth quartile is about $90 \%$.

respectively. A separate experiment (results not shown) confirmed that the results get better as the probabilities of being an attack source become more and more unequal.

\section{RELATED WORK}

Although there has been much research on attack tracing techniques, little work has been done on the issue of minimizing the number of monitors and optimizing their placement for the Internet. Several papers have identified this as a problem for future work, due to the issue of incremental deployment of new capabilities; see, for instance, [9].

One related work is due to Park and Lee [27]. In this work, the authors introduced a method for placement of filters that implement Route-based Distributed Packet Filtering (DPF). DPF was developed to prevent DDoS attacks using packets with spoofed source IP addresses. Park and Lee suggested a means of minimizing the number of ASes where packet filtering must be implemented, by heuristically solving the vertex cover problem. A vertex cover $V C$ of a graph $G(V, E)$ is defined as a subset of $V$ such that $\forall(u, v) \in E, u \in$ $V C$ or $v \in V C$. As a result, every non-filtering AS will be surrounded by ASes that implement filtering. This method was evaluated on an Internet AS topology and approximately $20 \%$ of the ASes were selected by their heuristic for the vertex cover. They showed that their vertex cover is within a factor of 2 of the optimal solution. Although the results can be compared with ours, the goals of their work are different from those of the monitor placement problem. First, the vertex cover does not allow adjustment of the desired maximum partition size, or ambiguity; it will always produce partitions of size 1, which may be inefficient and impractical. Second, the vertex cover is not designed for use as an edge separator; $100 \%$ of the edges are adjacent to at least one vertex in the cover, and would therefore be in the edge separator. Therefore, this approach is incapable of being applied when the optimization metric is edge cost rather than vertex cost. Their results on an Internet AS topology (from 1997, consisting of 3,015 ASes connected by 5,230 edges) required that approximately $20 \%$ of the ASes be monitored to achieve an exact partition size of 1 , compared
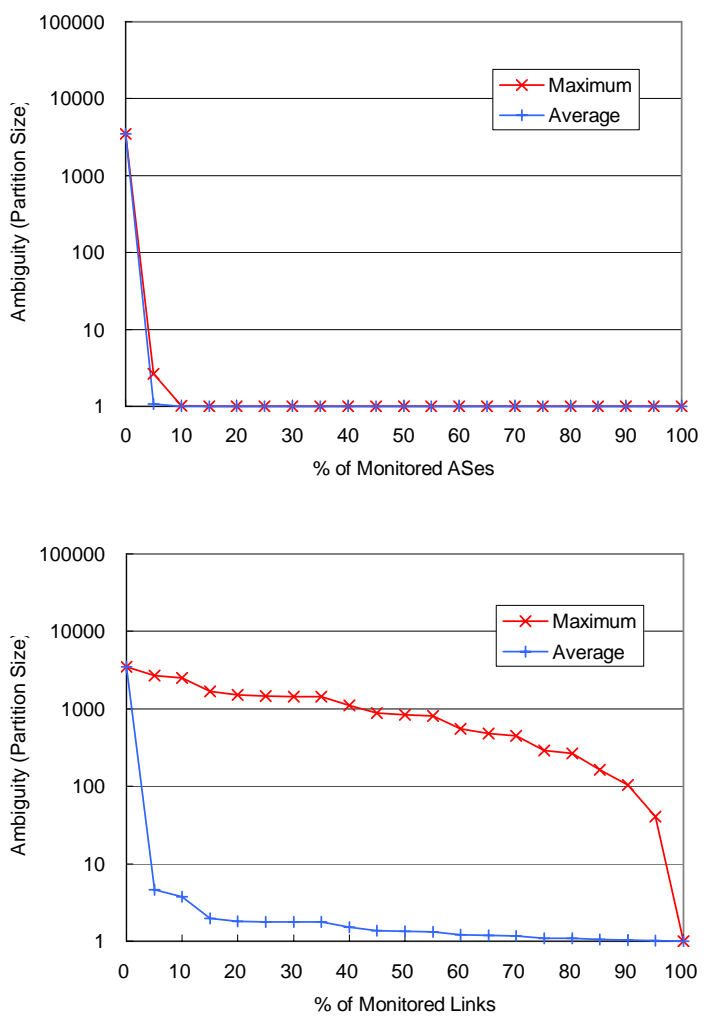

(b) Weighted edge separation for monitoring links.

Fig. 11. Maximum and average attribution ambiguity of the fourth quartile of ASes with an aggregate attack probability of $90 \%$.

with our heuristic's selection of 5\% of the ASes to achieve an average partition size close to 1 .

Another paper on monitor placement has been recently published by Tang et al. [28]. The authors addressed the monitor placement problem for attack attribution. Their goal was to find the optimal location of the monitors for a given number of monitors, which would minimize the entropy (uncertainty) in identifying the attack source after the completion of monitoring. The optimization process consists of a series of graph simplifications, which partitions the graph, and computation of the entropy of the resulting graphs. The graph is partitioned using greedy heuristics based on such factors as node degree, which is similar to our proposed vertex separator. The entropy of each partition of size $n$ was computed as $\log _{2} n$ where every node has equal probability of being the attack source. Then the worst case and average values over all partitions were computed. This method was evaluated on real network graphs with POP-level topologies, such as the AT\&T IP Backbone, DFN, Switch Network, etc. The results showed that the number of monitors required for a reasonable attribution performance ranged from $10 \%$ to $50 \%$ of the total number of nodes in the network. This is highly inefficient compared to our results for the AS-level graph, which requires less than $5 \%$ of the ASes to be monitored for similar precision. The networks used in [28] for evaluation of their method were quite small (less than 100 nodes), so comparison on 
large networks is not possible. In addition, this work addressed only vertex monitoring, and did not consider the issue of link monitoring.

\section{CONCLUSIONS}

In this paper we presented several approaches for strategic deployment of network monitors for attack attribution, independent of the attribution techniques. The goal is to minimize the number of monitors needed to achieve a desired degree of accuracy in attack attribution (or vice versa), and to determine the locations of those monitors. The problem was formulated as a graph partitioning problem. The intuition for defining the problem in this way is that it provides a very flexible metric for measuring quality, which is the partition size, or ambiguity in attributing attacks to the proper source. The experimental results showed that a degree-based heuristic for the separator works very well for the Internet topology with power-law connectivity, when the cost metric is the number of ASes selected for monitoring. When cost is measured by the number of links that must be monitored, a substantial fraction of the links must be monitored to achieve a reasonable degree of precision; an existing edge separator heuristic (METIS) performed well for the worst case ambiguity, while the proposed degree-based edge separator performed better for the average value. It was also demonstrated that the results are substantially improved using weighted separators, when ASes have different probabilities of being the source of an attack.

The proposed methods are somewhat static in nature, considering only the network topology. It is likely that dynamic approaches, using other information such as routing and intrusion detection information, would provide more promising results, in exchange for increased complexity and cost. Such techniques might require the ability to easily shift or move the monitoring capability as circumstances require. It remains to be seen if that is practical.

\section{REFERENCES}

[1] W. T. Strayer, C. E. Jones, I. Castineyra, J. B. Levin, and R. R. Hain, "An Integrated Architecture for Attack Attribution," BBN Technologies, 10 Moulton Street, Cambridge, MA 02138, Tech. Rep. BBN REPORT8384, Dec. 2003.

[2] L. T. Heberlein and M. Bishop, "Attack Class: Address Spoofing," in Proceedings of the 19th National Information Systems Security Conference (NISSC), Oct. 1996, pp. 371-377.

[3] T. Ylonen, "The Secure Shell (SSH) Protocol Architecture," IETF RFC: 4251, Jan. 2006. [Online]. Available: http://www.ietf.org/rfc/rfc4251.txt

[4] S. Kent, "Security Architecture for the Internet Protocol," IETF RFC: 2401, Nov. 1998. [Online]. Available: http://www.ietf.org/rfc/rfc2401.txt

[5] Y. Guan, X. Fu, D. Xuan, P. U. Shenoy, R. Bettati, and W. Zhao, "NetCamo: Camouflaging Network Traffic for QoS-Guaranteed Mission Critical Applications," IEEE Transactions on Systems, Man, and Cybernetics - Part A: Systems and Humans, vol. 31, no. 4, pp. 253-265, Jul. 2001.
[6] D. Goldschlag, M. Reed, and P. Syverson, "Onion Routing for Anonymous and Private Internet Connections," Communications of the ACM, vol. 42, no. 3, pp. 39-41, Feb. 1999.

[7] S. Staniford-Chen and L. T. Heberlein, "Holding Intruders Accountable on the Internet," in Proceedings of the 1995 IEEE Symposium on Security and Privacy (S\&P), May 1995, pp. 39-49.

[8] Y. Zhang and V. Paxson, "Detecting Stepping Stones," in Proceedings of the 9th USENIX Security Symposium, Aug. 2000, pp. 171-184.

[9] S. Savage, D. Wetherall, A. R. Karlin, and T. Anderson, "Practical Network Support for IP Traceback," in Proceedings of ACM SIGCOMM, Sep. 2000, pp. 295-306.

[10] A. C. Snoeren, C. Partridge, L. A. Sanchez, C. E. Jones, F. Tchakountio, B. Schwartz, S. T. Kent, and W. T. Strayer, "Hash-Based IP Traceback," in Proceedings of ACM SIGCOMM, Sep. 2001, pp. 3-14.

[11] M. T. Goodrich, "Efficient Packet Marking for Large-Scale IP Traceback," in Proceedings of the 9th ACM Conference on Computer and Communications Security (CCS), Oct. 2002, pp. 117-126.

[12] X. Wang and D. S. Reeves, "Robust Correlation of Encrypted Attack Traffic through Stepping Stones by Manipulation of Interpacket Delays," in Proceedings of the 10th ACM conference on Computer and Communications Security (CCS), Oct. 2003, pp. 20-29.

[13] Y. J. Pyun, Y. H. Park, X. Wang, D. S. Reeves, and P. Ning, "Tracing Traffic through Intermediate Hosts that Repacketize Flows," in Proceedings of the 26th IEEE Conference on Computer Communications (INFOCOM), May 2007.

[14] X. Wang, S. Chen, and S. Jajodia, "Tracking Anonymous Peer-to-Peer VoIP Calls on the Internet," in Proceedings of the 12th ACM conference on Computer and Communications Security (CCS), Nov. 2005, pp. 8191.

[15] R. Chawla, "Internet Exchange - A Solution Towards Cost Effective and Quality Internet Access," Global Internet Policy Initiative, 2002 [Online]. Available: http://www.internetpolicy.net/practices/

[16] "BGP - the Border Gateway Protocol. Advanced Internet Routing Resources." [Online]. Available: http://www.bgp4.as/

[17] C. Ashcraft and J. W. H. Liu, "A Partition Improvement Algorithm for Generalized Nested Dissection,” York University, Tech. Rep. BCSTECH-94-020, 1994

[18] A. Pothen, "Graph Partitioning Algorithms with Applications to Scientific Computing," in Parallel Numerical Algorithms, D. E. Keyes, A. Sameh, and V. Venkatakrishnan, Eds. Kluwer Academic Press, Jan. 1997, vol. 4, pp. 323-368.

[19] T. W. Haynes, S. T. Hedetniemi, and P. J. Slater, Fundamentals of domination in graphs. Marcel Dekker, 1998

[20] "CAIDA's Macroscopic Topology AS Adjacencies." [Online]. Available: http://www.caida.org/tools/measurement/skitter/as $\lfloor$ adjacencies.xml

[21] "CAIDA's Internet Topology Data Kit \#0304," cooperative Association for Internet Data Analysis, San Diego Supercomputer Center (SDSC), University of California, San Diego (UCSD), 2003.

[22] "University of Oregon's RouteViews Project." [Online]. Available: http://www.routeviews.org/

[23] C. Faloutsos, M. Faloutsos, and P. Faloutsos, "On Power-Law Relationships of the Internet Topology," in Proceedings of ACM SIGCOMM, Aug. 1999, pp. 251-262.

[24] B. W. Kernighan and S. Lin, "An Efficient Heuristic Procedure for Partitioning Graphs," Bell System Technical Journal, vol. 49, pp. 291307, 1970.

[25] G. Karypis and V. Kumar, "Multilevel k-way Partitioning Scheme for Irregular Graphs," Journal of Parallel and Distributed Computing, vol. 48, no. 1, pp. 96-129, Jan. 1998.

[26] Y. J. Pyun and D. S. Reeves, "Constructing a Balanced, $(\log (\mathrm{N}) / \log \log (\mathrm{N}))$-Diameter Super-Peer Topology for Scalable P2P Systems," in Proceedings of the 4th International Conference on Peer-to-Peer Computing (P2P), Aug. 2004, pp. 210-218.

[27] K. Park and H. Lee, "On The Effectiveness of Route-Based Packet Filtering for Distributed DoS Attack Prevention in Power-Law Internets," in Proceedings of ACM SIGCOMM, May 2001, pp. 15-26.

[28] Y. Tang, Y. Liverpool, and T. E. Daniels, "Monitor Placement for Stepping Stone Analysis," in Workshop on Information Assurance, Apr. 2006, pp. 505-512. 